\title{
Signaling Cost Analysis of Mobility Protocols using City Section Mobility Model
}

\author{
Md. Shohrab Hossain and Mohammed Atiquzzaman \\ School of Computer Science \\ University of Oklahoma, Norman, OK 73019 \\ Email: \{shohrab, atiq\}@ou.edu
}

\begin{abstract}
Increasing demand for mobility in wireless data network has given rise to various mobility management schemes. Most of the analysis on mobility protocols used Random Waypoint (RWP) mobility model which fails to represent realistic movement pattern. In this paper, we have used City Section Mobility (CSM) model, a realistic mobility model, to compare the signaling costs of two host-mobility protocols: HIMPv6 and SIGMA, and two network-mobility protocols: NEMO BSP and SINEMO. Our analysis shows that the signaling cost of SIGMA (SINEMO) is much less than that of HMIPv6 (NEMO BSP) for both mobility models. We further find that the lookup cost component, which is independent of mobility models, dominates the total signaling cost. Hence, the total signaling costs of mobility protocols do not vary much for different mobility models.
\end{abstract}

\section{INTRODUCTION}

Increasing demand for mobility in wireless data networks has given rise to various mobility management schemes. IETF proposed Mobile IPv6 [1] and Hierarchical Mobile IPv6 (HMIPv6) [2] to support host-mobility and NEtwork MObility Basic Support Protocol (NEMO BSP) [3] to support networkmobility. But these protocols have a number of drawbacks, such as, high handover latency, packet loss, inefficient routing path, and high signaling cost. To address these drawbacks, seamless IP diversity based host and network mobility protocols, SIGMA [4] and SINEMO [5] have been proposed.

In order to simulate movement of mobile nodes, mobility models are used. Examples of various mobility models are Random Walk, Random WayPoint (RWP), Random Direction, Gauss-Markov, and City Section. Previous research [6] has illustrated that the choice of a mobility model can significantly affect the performance evaluation of mobility protocols. Among these models, RWP model is the most common and frequently used model due to its simplicity. But it has a number of drawbacks as follows. In RWP model, a mobile node picks up a random speed and direction, resulting in sharp turns and sudden stops frequently which is very unusual in real scenarios. Moreover, the assumption of straight line movement is not always valid; there may be obstructions in its path. In contrast, City Section Mobility (CSM) model, introduced by Davies [6], [7], represents a movement behavior that is influenced by constraints of the environment. In real life scenarios, mobile nodes do not have the ability to travel freely; rather they have to follow traffic regulations, avoid obstacles,

This research work was supported by NASA Grant NNX06AE44G. buildings, etc. Thus CSM model is a realistic movement pattern for vehicles in a city.

Signaling is one of the major performance measures for mobility protocols. Protocols having high signaling traffic consume wireless bandwidth, resulting in inefficient throughput and increased packet delivery time. Most of the signaling cost analysis on mobility protocols [8], [9] have used RWP model. Our work differs from previous works by analyzing the realistic CSM model and using the model to compute the signaling cost of various host and network-mobility protocols.

The objective of this paper is to present a comprehensive analysis on the signaling costs of host and network-mobility protocols using a realistic mobility model, and to justify that SIGMA (SINEMO) requires less signaling cost than its IETF counterpart HMIPv6 (NEMO BSP).

Our contribution in this paper are (i) developing an analytical framework to compute subnet residence time for City Section mobility model, (ii) evaluating and comparing signaling cost between two host-mobility protocols, i.e., HMIPv6 and SIGMA, and (iii) evaluating and comparing signaling cost between two network-mobility protocols, i.e., NEMO BSP and SINEMO.

The rest of the paper is organized as follows. In Section II, the CSM model is analyzed. In Section III, a brief description of mobility protocols is given, followed by the expressions of signaling costs in Section IV. Section V presents the comparison on the signaling costs of these host and networkmobility protocols Finally, Section VI has the concluding remarks.

\section{City Section Mobility MOdel}

The simulation area used in CSM model [6], [7] is represented by a grid of streets forming a particular section of a city. The model sets the speed limit of each street. Each mobile node begins the simulation at a predefined intersection of two streets. It then randomly chooses a destination. Moving to this destination involves (at most) one horizontal and one vertical movement. Upon reaching the destination, it pauses for some random time and the same process is repeated. Each such cycle is termed as an epoch. Following are the assumptions of CSM model:

- Roads are parallel to axes.

- Starting point and each destination point are assumed to be road intersections. 


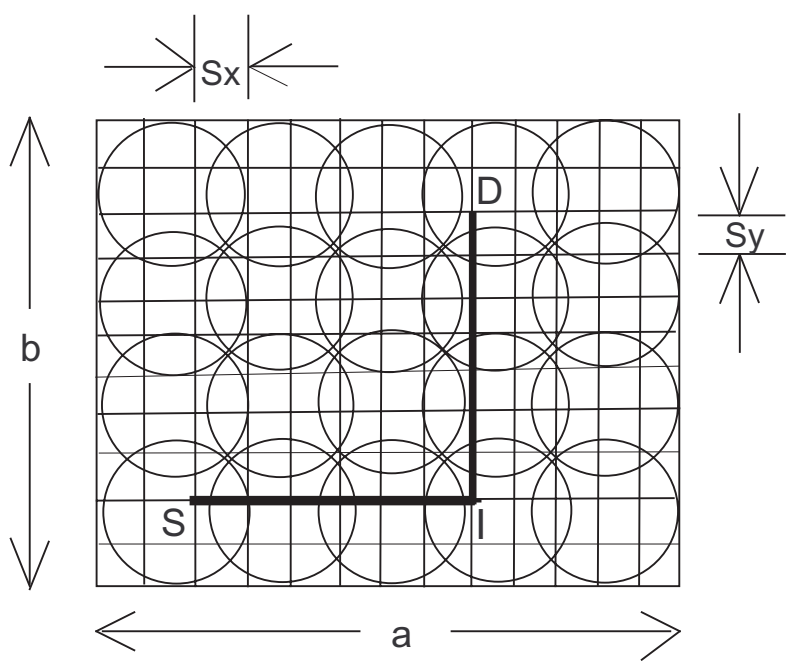

Fig. 1. Road network in CSM model.

Let the environment be a rectangular area of dimension $a \times b$ as shown in Fig. 1. Let there be $N_{s}$ horizontal roads (streets) and $N_{a}$ vertical roads (avenues) and streets be $S_{y}$ distance apart and avenues be $S_{x}$ distance apart. So number of streets are $N_{a}=a / S_{x}+1$, and number of avenues are, $N_{s}=b / S_{y}+$ 1.

\section{A. Expected Epoch Length}

Let us number each of the avenues as $1,2, \ldots, N_{a}$ from left to right and each of the streets as $1,2, \ldots, N_{s}$ from top to bottom. Let us first find out the expected length that a Mobile Node travels in horizontal direction in an epoch. Let $L_{x}$ be the length of one instance. The values of $L_{x}$ can be found from a $N_{a} \times N_{a}$ matrix, each of whose entry is given by,

$$
M(i, j)=S_{x}|i-j|, \text { where } 1 \leq i, j \leq N_{a}
$$

So the values of $L_{x}$ can be $0, S_{x}, 2 S_{x}, \ldots,\left(N_{a}-1\right) S_{x}$ depending on the location of points $S$ and $I$. Hence, the expected value of $L_{x}$ is given by,

$$
\begin{aligned}
E\left(L_{x}\right)= & \frac{1}{N_{a}{ }^{2}}\left[N_{a} \times 0+2\left\{\left(N_{a}-1\right) S_{x}+\left(N_{a}-2\right) 2 S_{x}\right.\right. \\
& \left.\left.+. .+2\left(N_{a}-2\right) S_{x}+1\left(N_{a}-1\right) S_{x}\right\}\right] \\
= & \frac{2 S_{x}}{N_{a}{ }^{2}} \sum_{i=1}^{N_{a}-1}\left(N_{a}-i\right) i \\
= & \frac{a\left(N_{a}+1\right)}{3 N_{a}}
\end{aligned}
$$

Similarly, for vertical movement, $E\left(L_{y}\right)=b\left(N_{s}+1\right) / 3 N_{s}$. Therefore, the expected epoch length can be obtained as

$$
E(L)=\frac{a\left(N_{a}+1\right)}{3 N_{a}}+\frac{b\left(N_{s}+1\right)}{3 N_{s}}
$$

For large values of $N_{a}$ and $N_{s}, E(L)=a / 3+b / 3$.

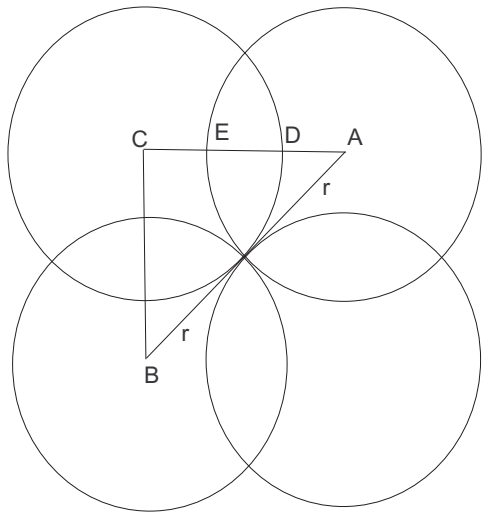

Fig. 2. Subnet Overlapping in CSM model.

\section{B. Epoch Time}

Let the speed of the mobile node vary between $V_{x}^{\min }$ to $V_{x}^{\max }$ for some horizontal road segment. So for uniform speed distribution, its probability density function is given by

$$
f_{V}\left(v_{x}\right)=\frac{1}{V_{x}^{\max }-V_{x}^{\min }}
$$

The expected time required for horizontal movement in an epoch is thus,

$$
\begin{array}{r}
E\left(T_{x}\right)=E\left(L_{x}\right) \int_{V_{x}^{\min }}^{V_{x}^{\max }} \frac{1}{v_{x}} f_{V}\left(v_{x}\right) d v_{x} \\
=E\left(L_{x}\right) \frac{\ln \left(V_{x}^{\max } / V_{x}^{\text {min }}\right)}{V_{x}^{\text {max }}-V_{x}^{\text {min }}}
\end{array}
$$

Similarly, for movement on vertical road segment

$$
E\left(T_{y}\right)=E\left(L_{y}\right) \frac{\ln \left(V_{y}^{\max } / V_{y}^{\min }\right)}{V_{y}^{\text {max }}-V_{y}^{\text {min }}}
$$

Adding Eqns. (4) and (5), the expected epoch time can be found as,

$$
E(T)=E\left(T_{x}\right)+E\left(T_{y}\right)
$$

\section{Pause Time}

In order to avoid collisions at each road intersection, there is a random pause time between 0 to $U_{\max }$. So the mean pause time is $E(U)=U_{\max } / 2$

\section{Number of Subnet Crossing}

Let us consider that the road network of dimensions $a \times b$ (Fig. 1) is covered by Access Points (AP); let there be $n$ rows of APs and $m$ APs in each row. In total, there will be $m n$ APs to cover the rectangular area. Let the radio coverage area of each AP be a circular region of radius $r$ and two successive APs overlap at a maximum length of $l$ along its diameter. So

$$
\begin{gathered}
a=2 r m-(m-1) l \\
b=2 r n-(n-1) l
\end{gathered}
$$

Let the radius $r$ of each subnet be greater than the inter-road spacing, i.e., $r>S_{x}$ and $r>S_{y}$. Let us assume that $2 r=$ $K S_{x}=K S_{y}$. In Fig. 2, the length of $A B=2 r$, and let $A C=$ 
$x=B C, D E=l$. Since the cells are parallel to axes, we find that $x=\sqrt{2} r=r+r-l$. Hence, $l=(2-\sqrt{2}) r$ Now putting the value of $l$ in Eqn. (7), we get, $m=\left[\frac{\sqrt{2} a-2(\sqrt{2}-1) r}{2 r}\right]$. Similarly, we can have $n=\left[\frac{\sqrt{2} b-2(\sqrt{2}-1) r}{2 r}\right]$. For $a=36 \mathrm{~km}$, $b=24 \mathrm{~km}$, and $r=0.5 \mathrm{~km}$, we find $m=51$, and $n=34$.

Now for movement along horizontal direction, if the distance between the two endpoints is less than the diameter of an AP's coverage area, there will be at most one subnet crossing. For any distance between $K S_{x}$ to $(2 K-1) S_{x}$, there will be at most two subnet crossings, and so on. Thus we can find out the expected number of subnet crossings in an epoch for movement along horizontal direction as,

$$
\begin{aligned}
E\left(C_{x}\right) & =\frac{2}{N_{a}^{2}}\left[\left(N_{a}-1\right)+\left(N_{a}-2\right)+. .+N_{a}-(K-1)\right. \\
& +2\left\{\left(N_{a}-K\right)+. .+\left(N_{a}-2 K+1\right)\right\}+. .+ \\
& \left.m\left\{\left(N_{a}-(m-1) K\right)+. .+\left(N_{a}-m K+1\right)\right\}\right] \\
& =\frac{2}{N_{a}^{2}}\left[K N_{a} \sum_{i=1}^{m} i-K^{2} \sum_{i=2}^{m} i(i-1)-\sum_{i=1}^{K-1} i \sum_{j=1}^{m} j\right] \\
& =\frac{m(m+1) K}{6 N_{a}{ }^{2}}\left(6 N_{a}-4 m K+K+3\right)
\end{aligned}
$$

Similarly, for movement along vertical direction

$$
E\left(C_{y}\right)=\frac{n(n+1) K}{6 N_{s}^{2}}\left(6 N_{s}-4 n K+K+3\right)
$$

The expected number of subnet crossing in an epoch is thus

$$
E(C)=E\left(C_{x}\right)+E\left(C_{y}\right)
$$

\section{E. Subnet Residence Time}

Since in each epoch, the mobile node pauses at two different points, the average residence time in a subnet can be estimated as follows:

$$
T_{r}=\frac{E(T)+2 E(U)}{E(C)}
$$

A similar analysis on RWP model is done in [10] to compute $T_{r}$. These expressions of subnet residence time $\left(T_{r}\right)$ will be used to compute the signaling costs in Section V.

\section{Mobility Protocols}

In this section, we give a brief description of two hostmobility protocols: SIGMA and HMIPv6, and two networkmobility protocols: SINEMO and NEMO BSP.

\section{A. Hierarchical Mobile IPv6}

Enhancement to MIPv6 [1] has resulted in Hierarchical Mobile IPv6 (HMIPv6) [2] where a new network element, called Mobility Anchor Point (MAP), is used to introduce hierarchy in mobility management. A MAP, essentially a local Home Agent, covers several subnets, called a region. A Mobile Host (MH) entering a MAP domain receives Router Advertisements containing information on one or more local MAPs. The MH updates the Home Agent (HA) with an address assigned by the MAP, called Regional CoA (RCoA), as its current location. The MAP intercepts all packets sent to $\mathrm{MH}$, encapsulates, and forwards them to MH's current address.

\section{B. SIGMA}

SIGMA [4] utilizes IP-diversity to achieve a seamless handover of a mobile host (MH), and is designed to solve many of the drawbacks of Mobile IP. The Location Manager (LM) is responsible for keeping location database of mobile hosts. Whenever any Correspondent Node $(\mathrm{CN})$ wants to send data to a MH, it must first send a query message to the LM to obtain its current IP address. For this, every MH must send its new IP address in a network it has moved to the LM; these are termed as Location Updates. Moreover, every subnet crossing triggers binding updates. Each MH needs to send a binding update after handover to every $\mathrm{CN}$ it is communicating with.

\section{NEMO BSP}

In NEMO BSP [3], Mobile Router (MR) ensures connectivity of all hosts inside the Mobile Network (MN) when MR changes its point of attachment to the Internet while moving from a Home Network to a Foreign Network (FN). An MR has its unique IP address and one or more mobile network prefixes that it advertises to the MHs attached to it. MR establishes a bidirectional tunnel with its HA to pass all traffic between its MHs and the CNs. When MR changes its point of attachment, it acquires a new care-of-address from the visited FN. It sends a Binding Update to its HA that creates a cache entry, binding MRs home address with its care-of-address, and creates a bidirectional tunnel between HA and MR.

\section{SINEMO}

SINEMO [5] uses the concept of SIGMA for seamless handoff. Here, MR acts as a gateway between the hosts and the APs for Internet access. When a mobile network moves into one subnet, MR obtains its own public IP address and one or more address prefixes. MR provides each host with a private IP address and reserves a public IP address for it. After handover, only the public addresses are modified in the address mapping at MR, the private IP addresses of the hosts remain unchanged. MR thus hides mobility from the hosts.

\section{Signaling Costs of Mobility Protocols}

In this section, we discuss the signaling costs of SIGMA, HMIPv6, SINEMO and NEMO BSP.

\section{A. Signaling Cost of SIGMA}

Every subnet crossing of a $\mathrm{MH}$ that happens in every $T_{r}$ seconds triggers a location update message to LM, and binding updates to every $\mathrm{CN}$, with which the $\mathrm{MH}$ is communicating. In addition, some location database lookup and tunneling are required at LM. Hence, the signaling cost of SIGMA [8] consists of three components: location update cost $\left(\Psi_{S G}^{L U}\right)$, binding update cost $\left(\Psi_{S G}^{B U}\right)$, and lookup cost $\left(\Psi_{S G}^{L U P}\right)$.

$$
\Psi_{S G}^{L U}=N_{m h} \frac{2\left(l_{m l}-1+\rho\right) \delta_{L}+\gamma_{l}}{T_{r}}
$$




$$
\begin{gathered}
\Psi_{S G}^{B U}=N_{m h} N_{c n} \frac{2\left(l_{m c}-1+\rho\right) \delta_{B}}{T_{r}} \\
\Psi_{S G}^{L U P}=N_{m h}^{2} N_{c n} \frac{\psi \lambda_{s}}{S}
\end{gathered}
$$

$N_{m h}=$ number of Mobile Hosts,

$N_{c n}=$ average number of $\mathrm{CN}$ communicating,

$\delta_{L}, \delta_{B}=$ per hop message transmission cost for location update and binding update, respectively,

$\rho=$ proportionality constant of signaling cost over wired and wireless link, $\psi=$ linear coefficient for lookup cost,

$l_{m l}=$ average number of hops between $\mathrm{MH}$ and LM,

$l_{m c}=$ average number of hops between MR and $\mathrm{CN}$,

$\gamma_{l}=$ processing cost at LM

$S=$ number of sessions,

$\lambda_{s}=$ average session arrival rate

\section{B. Signaling Cost of HMIPv6}

In HMIPv6, every subnet crossing within the MAP (happens every $T_{r}$ seconds) triggers a registration to the MAP, and for every region crossing between MAPs (happens every $M \times T_{r}$ seconds), MH needs to register with HA. In addition, some location database lookup and tunneling are required at MAP and HA. No binding update is sent to CNs from MHs. Hence, the signaling cost of HMIPv6 [8] consists of two components: location update cost $\left(\Psi_{H M}^{L U}\right)$ and lookup cost $\left(\Psi_{H M}^{L U P}\right)$.

$$
\begin{array}{r}
\Psi_{H M}^{L U}=N_{m h}\left[\frac{2\left(l_{m m}-1+\rho\right) \delta_{L}+\gamma_{m}}{T_{r}}+\right. \\
\left.\frac{2\left(l_{m m}+l_{m h}-1+\rho\right) \delta_{L}+\gamma_{h}+2 \gamma_{m}}{T_{r}} \times \frac{m n-R}{2 m n-R-1}\right] \\
\Psi_{H M}^{L U P}=N_{m h} N_{c n} \lambda_{s}\left(\psi N_{m h} \frac{m n+R}{m n}+3 \tau\right)
\end{array}
$$

$l_{m h}=$ average distance between MAP and HA in hops,

$l_{m m}=$ average distance between MH and MAP in hops,

$\gamma_{h}, \gamma_{m}=$ processing cost for each location update at HA and MAP, respectively, $\tau=$ encapsulation cost,

$m=$ number of APs in a row,

$n=$ number of APs in a column,

$R=$ number of subnets under a MAP

\section{Signaling Cost of SINEMO}

In SINEMO, signaling is incurred due to MR movement out of a subnet, $\mathrm{MH}$ movement within $\mathrm{MN}$, and for binding updates to be sent to CNs. Thus the signaling cost of SINEMO [9] consists of three components: location update cost $\left(\Psi_{S N}^{L U}\right)$, binding update cost $\left(\Psi_{S N}^{B U}\right)$, and lookup cost $\left(\Psi_{S N}^{L U P}\right)$. Here we consider the residence time for $\mathrm{MR}$ and $\mathrm{MH}$ to be equal.

$$
\begin{gathered}
\Psi_{S N}^{L U}=\frac{N_{m h}\left(2 \rho \delta_{L}+2 \gamma_{l}\right)+N_{m r}\left(2\left(l_{r l}-1+\rho\right) \delta_{L}+\gamma_{l}\right)}{T_{r}} \\
\Psi_{S N}^{B U}=N_{m h} N_{c n} \frac{2\left(2 l_{m c}-4+3 \rho\right) \delta_{B}-2}{T_{r}}
\end{gathered}
$$

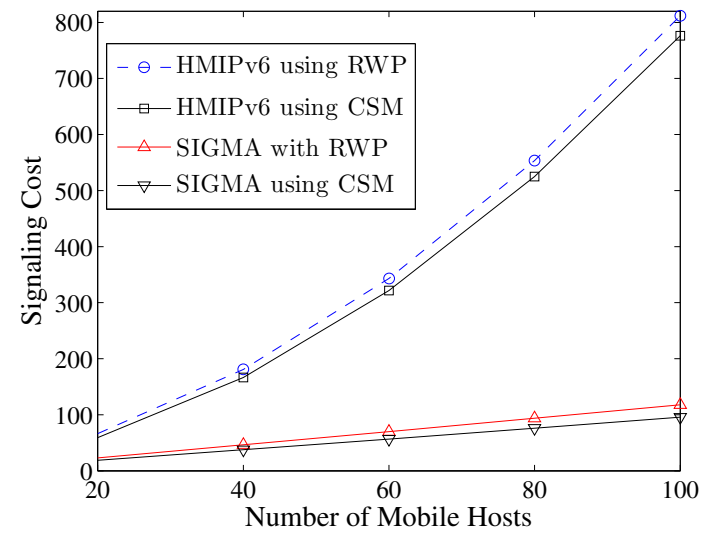

Fig. 3. Signaling cost of HMIPv6 and SIGMA vs. number of MHs using RWP and CSM model.

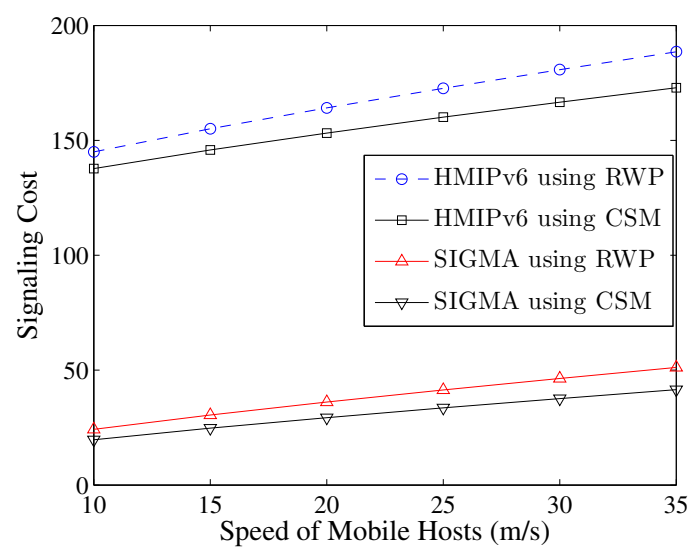

Fig. 4. Signaling cost of HMIPv6 and SIGMA vs. Speed of MH using RWP and CSM model.

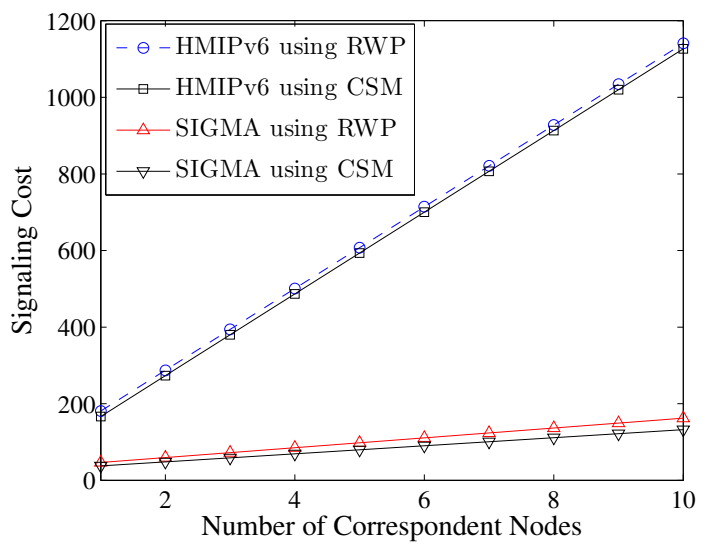

Fig. 5. Signaling cost of HMIPv6 and SIGMA vs. number of CNs using RWP and CSM model. 


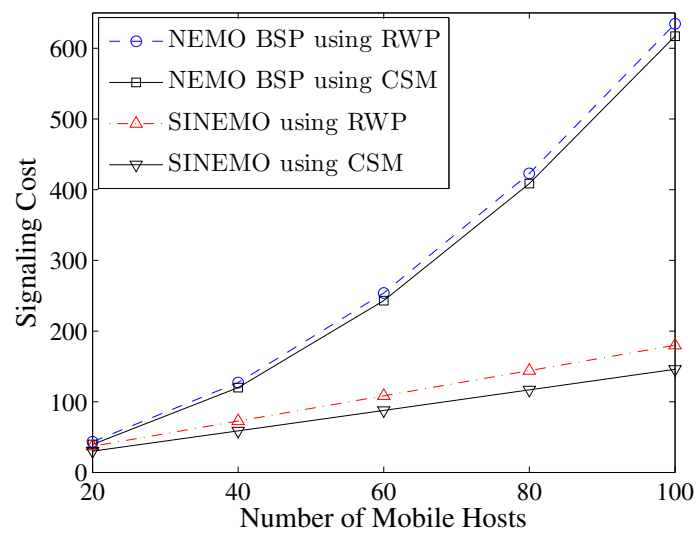

Fig. 6. Signaling cost of NEMO BSP and SINEMO vs. number of MHs using RWP and CSM model.

$$
\Psi_{S N}^{L U P}=\omega N_{m h}^{2} N_{c n} \frac{\psi \lambda_{s}}{S}
$$

$N_{m r}=$ number of mobile routers,

$l_{r l}, l_{m c}=$ average number of hops between MR and CLM, $\mathrm{MR}$ and $\mathrm{CN}$, respectively

$\gamma_{l}, \gamma_{h}, \gamma_{r}=$ processing cost at LM (SINEMO), at HA-H and HA-M (NEMO BSP), respectively

$\omega=$ ratio of MHs that are servers to total $\mathrm{MH}$,

$P=$ maximum transmission unit, $F=$ filesize.

\section{Signaling Cost of NEMO BSP}

Unlike SINEMO, no binding update is sent to CNs in NEMO BSP. So its signaling cost consists of two components: location update $\left(\Psi_{B S P}^{L U}\right)$ and lookup cost $\left(\Psi_{B S P}^{L U P}\right)$ [9].

$$
\begin{array}{r}
\Psi_{B S P}^{L U}=\left[N_{m h}\left(\left(2 l_{m h}-2+2 \rho\right) \delta_{L}+\gamma_{h}\right)+\right. \\
\left.N_{m r}\left(2\left(l_{m r}-1+\rho\right) \delta_{L}+\gamma_{r}\right)\right] / T_{r} \\
\Psi_{B S P}^{L U P}=N_{m h} N_{c n} \lambda_{s} \frac{F}{P}\left(\psi N_{m h}+\tau\right)
\end{array}
$$

\section{NuMERIC RESUlts}

In this section, we compare host-mobility protocols ( $\mathrm{Sec}$ $\mathrm{V}-\mathrm{A}$ ) and network-mobility protocols (Sec V-B) using CSM and RWP model. The comparison with RWP model is based on the analysis presented in [10] which assumes that the coverage area of each AP is square-shaped that is not realistic. For the sake of comparison, we are assuming the dimensions of each AP's coverage area of RWP model to be $2 r \times 2 r$.

Parameters relating to mobility models are: $a=36 \mathrm{~km}, b=$ $24 \mathrm{~km}, S_{x}=200 \mathrm{~m}, S_{y}=200 \mathrm{~m}, K=5, V_{x}^{\max }=V_{y}^{\max }=120 \mathrm{~km}$ $/ \mathrm{hr}, V_{x}^{\text {min }}=V_{y}^{\text {min }}=25 \mathrm{~km} / \mathrm{hr}$, and $E(u)=4 \mathrm{sec}$. Parameters relating to mobility protocols are similar as the previous works [8], [9]: $\delta_{L}=\delta_{B}=0.2, \rho=10, \lambda_{s}=0.01, \gamma_{l}=\gamma_{h}=30, \gamma_{r}=$ $1.5 \times \gamma_{h}, N_{c n}=1, R=10, l_{m l}=l_{m c}=l_{m h}=l_{r l}=l_{m r}=35$, $\tau=0.5, S=10, \omega=0.5, \mathrm{~F}=10 \mathrm{~Kb}$, and $\mathrm{P}=576 \mathrm{~b}$.

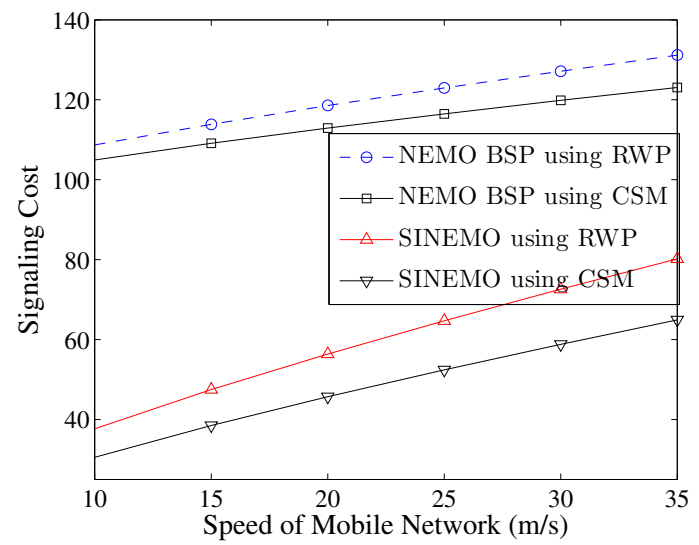

Fig. 7. Signaling cost of NEMO BSP and SINEMO vs. Speed of MN using RWP and CSM model.

\section{A. Host-Mobility Protocols}

This section presents the results of two host-mobility protocols: HMIPv6 and SIGMA. Fig. 3 shows the impact of the number of mobile hosts on signaling costs of HMIPv6 and SIGMA for both CSM and RWP models. Results show that the signaling cost of SIGMA is much less than that of HMIPv6 for both mobility models.

Fig. 4 shows the impact of $\mathrm{MH}$ speed on signaling costs of HMIPv6 and SIGMA for CSM and RWP models. Here we use $N_{m h}=40$. Results show that the signaling cost of SIGMA is much less than that of HMIPv6 for both mobility models.

Fig. 5 shows the impact of number of $\mathrm{CNs}$ on signaling costs of HMIPv6 and SIGMA for both mobility models with $N_{m h}$ $=40$ and speed of $30 \mathrm{~m} / \mathrm{s}$. Here, we also find that signaling cost of HMIPv6 is much higher than that of SIGMA for both mobility models.

\section{B. Network-Mobility Protocols}

This section presents results of two network-mobility protocols: NEMO BSP and SINEMO. Fig. 6 shows the impact of the number of mobile hosts on signaling costs of NEMO BSP and SINEMO for both mobility models. Results show that the signaling cost of SINEMO is much less than that of NEMO BSP for both mobility models.

Fig. 7 shows the signaling cost for NEMO BSP and SINEMO using both RWP and CSM model for varying speed of MN with $N_{m h}=40$. Fig. 8 shows the the impact of number of CNs on signaling costs of NEMO BSP and SINEMO using both mobility models with $N_{m h}=40$ and speed $=30 \mathrm{~m} / \mathrm{s}$. Fig. 9 shows the the impact of number of Mobile Routers on signaling costs of NEMO BSP and SINEMO using both mobility models with $N_{m h}=40$ and speed $=30 \mathrm{~m} / \mathrm{s}$. In all these cases, the signaling costs of SINEMO are found to be much less than that of NEMO BSP for both mobility models.

From the above graphs (see Figs. 3 - 9), we found that two different mobility models (CSM and RWP) produce almost similar signaling graphs. This is due to the existence of a dominant term (lookup cost) in the total signaling cost and this lookup cost is independent of mobility models. Other than 


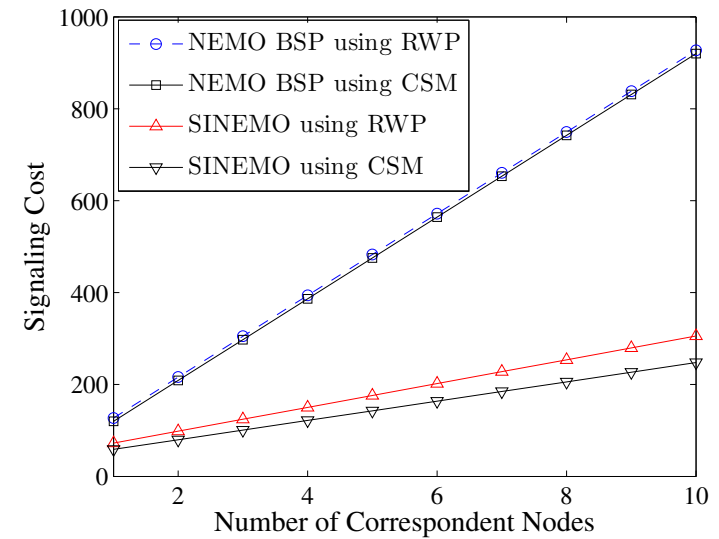

Fig. 8. Signaling cost of NEMO BSP and SINEMO vs. number of CNs using RWP and CSM model.

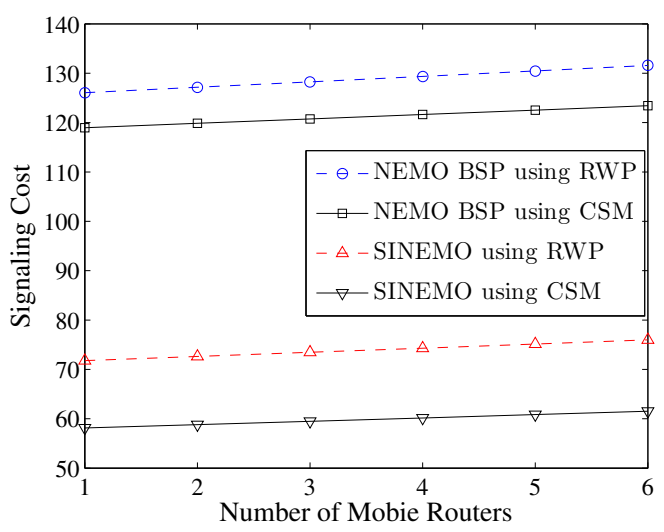

Fig. 9. Signaling cost of NEMO BSP and SINEMO vs. number of MRs using RWP and CSM model.

this term, the location update cost of the mobility protocols varies much for different mobility models. This is evident from the following two graphs (Fig. 10 and 11) which show the location update costs only. In Fig. 10, we see that the location update cost of HMIPv6 differs much for RWP and CSM model. Similar statement holds for SIGMA, and also for NEMO and SINEMO (Fig. 11).

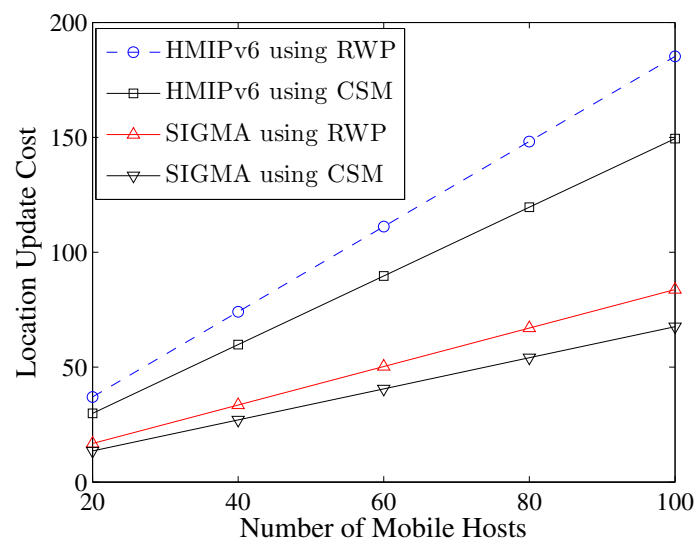

Fig. 10. Location Update cost of HMIPv6 and SIGMA vs. number of MHs using RWP and CSM model.

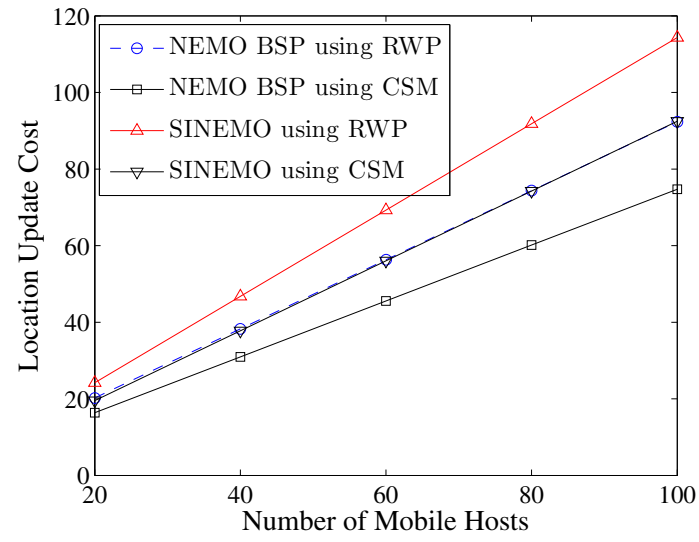

Fig. 11. Location Update cost of NEMO BSP and SINEMO vs. number of MHs for RWP and CSM model.

\section{CONCLUSION}

In this paper, we have analyzed the CSM model to compute its subnet residence time and use it and RWP model to find out the signaling costs of various mobility protocols. We have presented numerical results for host-mobility protocols (HMIPv6 vs. SIGMA) and network mobility protocols (NEMO BSP vs. SINEMO) based on number of mobile hosts, speed of mobile hosts, number of correspondent nodes and number of mobile routers. It is found that SIGMA (SINEMO) requires much less signaling than its IETF counterpart HMIPv6 (NEMO BSP). We also find that the highly dominant lookup cost, which is independent of mobility models, makes the total signaling costs to be almost similar in spite of the location update cost being different. This leads to an interesting conclusion that the two different mobility models (RWP and CSM) produce almost similar signaling cost.

\section{REFERENCES}

[1] D. Johnson, C. E. Perkins, and J. Arkko, "Mobility support in IPv6," IETF RFC 3775, June 2004

[2] H. Soliman, C. Castelluccia, K. E. Malki, and L. Bellier, "Hierarchical Mobile IPv6 mobility management (HMIPv6)," IETF RFC 4140, Aug 2005.

[3] V. Devarapalli, R. Wakikawa, A. Petrescu, and P. Thubert, "NEtwork MObility (NEMO) basic support protocol," RFC 3963, Jan 2005.

[4] S. Fu and M. Atiquzzaman, SIGMA: A Transport Layer Handover Protocol for Mobile Terrestrial and Space Networks, ser. e-Business and Telecommunication Networks. Springer, 2006.

[5] P. Chowdhury, M. Atiquzzaman, and W. Ivancic, "SINEMO: An IPdiversity based approach for network mobility in space," in Second IEEE International Conference on Space Mission Challenges for Information Technology (SMC-IT), Pasadena, CA, July 17-21, 2006, pp. 109-115.

[6] T. Camp, J. Boleng, and V. Davies, "A survey of mobility models for ad hoc network research," Wireless Communications and Mobile Computing, vol. 2, pp. 483-502, 2002.

[7] V. Davies, "Evaluating mobility models within an Ad Hoc Network," MS thesis, Colorado School of Mines, 2000.

[8] S. Fu and M. Atiquzzaman, "Signaling cost and performance of SIGMA: A seamless handover scheme for data networks," Wireless Communication and Mobile Computing, vol. 5, no. 7, pp. 825-845, Nov 2005.

[9] A. S. Reaz, P. K. Chowdhury, and M. Atiquzzaman, "Signaling cost analysis of SINEMO: Seamless End-to-End Network Mobility," in First ACM/IEEE International Workshop on Mobility in the Evolving Internet Architecture, San Francisco, CA, Dec 01, 2006, pp. 37-42.

[10] C. Bettstetter, H. Hartenstein, and X. Prez-Costa, "Stochastic properties of Random Waypoint mobility model," Wireless Networks, vol. 10, no. 5, pp. 555-567, Sept. 2004. 